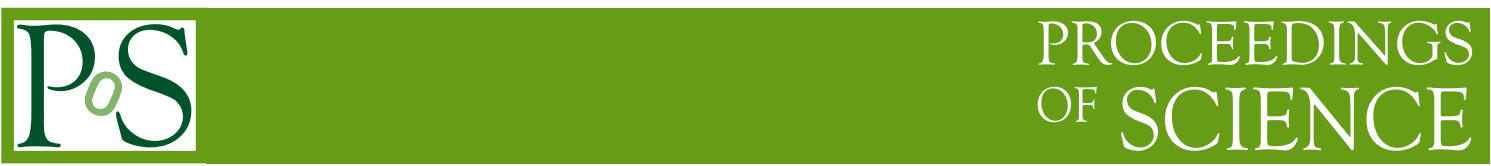

\title{
Gravitational lensing by gravitational wave pulse
}

\author{
G.S. Bisnovatyi-Kogan ${ }^{a b c}$ and O.Yu. Tsupko ${ }^{* a c}$ \\ ${ }^{a}$ Space Research Institute of Russian Academy of Science, \\ Profsoyuznaya 84/32, Moscow 117997, Russia \\ ${ }^{b}$ Joint Institute for Nuclear Research, Dubna, Russia \\ ${ }^{c}$ Moscow Engineering Physics Institute, Moscow, Russia \\ E-mail: gkogan@iki.rssi.ru, tsupko@iki.rssi.ru
}

Gravitational lensing by gravitational wave is considered. We have found that although final and initial directions of photons after passing through the gravitational wave pulse coincide, the gravitational wave changes the photon propagation, simply shifting its whole trajectory. This displacement in the trajectory is found analytically for the photon passing through the plane gravitational wave pulse. On the basis of this result we obtain an approximate formula for the estimation of observational effects.

The Manchester Microlensing Conference: The 12th International Conference and ANGLES Microlensing Workshop

January 21-25 2008

Manchester, $U K$

* Speaker. 


\section{Introduction}

Gravitational lensing by gravitational wave (GW) in different cases was considered by many authors (see [1], [2], [3] and references therein). It was found that the deflection angle vanishes for any localized GW packet because of transversality of GW [3]. Thus if the photon passes through finite wave pulse its deflection due to this wave is equal to zero.

In this work we confirm analytically vanishing of deflection angle for plane wave pulses. However, we have found that the GW changes the photon propagation in another way, simply shifting its whole trajectory after passing through the GW pulse (see Fig.1). This displacement is found analytically for the photon passing through the plane GW pulse (see also [4]). On the basis of this result we obtain an approximate formula for the estimation of observational effects.

\section{The deflection angle}

The wave vector of the photon $k^{i}$ (tangent to a trajectory) by definition is equal to $k^{i}=\frac{d x^{i}}{d \lambda}$, where $\lambda$ is a parameter changing along the trajectory [5]. The geodesic equation is written as $D k^{i}=0$ or $D k_{i}=0$, where $D$ denotes a covariant derivative. After some transformation (see [4] for details) we obtain the equation of motion for the photon:

$$
\frac{d k_{i}}{d \lambda}=\frac{1}{2} k^{k} k^{l} \frac{\partial g_{k l}}{\partial x^{i}}, \quad \text { or } \ddot{x}_{i}=\frac{1}{2} \dot{x}^{k} \dot{x}^{l} \frac{\partial g_{k l}}{\partial x^{i}},
$$

where dot denotes derivative with respect to parameter $\lambda$.

Let us consider the GW in a flat space with a metric $g_{i k}=\eta_{i k}+h_{i k}, h_{i k} \ll 1$, where $\eta_{i k}$ is a flat metric $(-1,1,1,1)$ and $h_{i k}$ is a small perturbation $(\mathrm{GW})$. In this approximation one can integrate equation (2.1), calculating right-hand side of equation with unperturbed trajectory of the photon. Performing integration, we obtain the expression for the deflection angle (compare with [3]):

$$
\hat{\alpha}_{i}=\frac{k_{i}(+\infty)-k_{i}(-\infty)}{k}=\int_{-\infty}^{+\infty} \frac{1}{2} k^{k} k^{l} \frac{\partial h_{k l}}{\partial x^{i}} d \lambda
$$

where $h_{i k}$ is calculated along the straight line trajectory and $k^{i}=$ const along unperturbed trajectory.

Consider the photon moving along $z$-axis. Its unperturbed trajectory is $z=z_{0}+c t$, and we can use the coordinate $z$ as the parameter $\lambda$. Then the wave vector is $k^{i}=(1,0,0,1), k=1$. When the photon passes through the finite wave packet, we denote the $z$-coordinate of the input of the photon into the wave front as $z_{1}$ and the $z$-coordinate of the output from the wave front as $z_{2}\left(z_{1}<z_{2}\right)$. Hence we have the expression for the deflection angle in the form (compare with [2]):

$$
\hat{\alpha}_{i}=\frac{1}{2} \int_{z_{1}}^{z_{2}} \frac{\partial}{\partial x^{i}}\left(h_{00}+2 h_{03}+h_{33}\right) d z .
$$

Let us calculate photon deflection by the plane GW pulse. Let us consider a light ray propagating under the angle $\varphi=-(\pi-\theta)$ relative to the direction of the plane $\mathrm{GW}$ packet propagation (see Fig.1a). Let us define for convenience two reference systems $K$ and $K^{\prime}$. The first one is connected with direction of the light ray: the photon moves along $z$-axis in a positive direction in the 
reference frame $K$. The second one is connected with the direction of propagation of the GW pulse. The GW packet moves along $z^{\prime}$-axis in a positive direction in the reference frame $K^{\prime}$ (see Fig.1a). The systems are at the rest relative to each other and their origins of coordinates coincide. The reference system $K$ transforms into the system $K^{\prime}$ by rotation by the angle $\varphi=-(\pi-\theta)$ around the axis $x$ (positive rotation is anticlockwise). At the initial time $t=0$ the photon is situated at $z_{0}<0\left(x_{0}=y_{0}=0\right)$, the wave vector of the photon is $k^{i}=\left(k^{0}, 0,0, k^{z}\right)=(1,0,0,1), k=k^{z}$. The form of the wave pulse is sinusoidal (the top part of the sinusoid, with the phase changing from 0 to $\pi$, and with zero perturbations on the boundaries):

$$
h_{i k}^{\prime} \propto \sin \xi^{\prime}, \quad \xi^{\prime}=0 . . \pi, \quad \xi^{\prime}=\omega t-k_{g}^{\prime} z^{\prime}, \quad k_{g}^{\prime}=\omega / c,
$$

where $\omega$ and $k_{g}^{\prime}$ are the frequency and the wave vector of GW in $K^{\prime}$ correspondingly. The pulse width $\delta$ (in space) is $\delta=c \pi / \omega$. At the initial time $t=0$ the GW pulse is situated at $-\delta<z^{\prime}<0$. It is convenient to use non-dimensional variables for time $\tilde{t}=t / t_{0}, t_{0}=1 / \omega$ and distances $\tilde{x}=x / x_{0}$, $x_{0}=c / \omega$. Hereafter we omit tildes for simplicity. In non-dimensional variables the equation (2.1) looks the same, and the GW form is written as $\sin \left(t-z^{\prime}\right)$.

The right side of (2.3) includes components $h_{00}, h_{03}, h_{33}$, which are components of gravitational perturbation in the reference system $K$. GW moves along the axis $O z^{\prime}$ in the reference system $K^{\prime}$, therefore the GW has non-zero components $h_{11}^{\prime}, h_{12}^{\prime}, h_{21}^{\prime}, h_{22}^{\prime}$ only. In the reference system $K$ we have $h_{00}=h_{03}=0, h_{33}=\sin ^{2} \varphi h_{22}^{\prime}$. Writing $h_{22}^{\prime}$ as $h_{22}^{\prime}=h \sin \left(t-z^{\prime}\right)$, where $h$ is the amplitude of wave, we obtain:

$$
h_{33}=h \sin ^{2} \theta \sin (t+z \cos \theta-y \sin \theta) .
$$

Taking into account that the straight line ray has the trajectory $z=z_{0}+t$, one can find the points of intersection of the photon and the wave front. The point of the input is $z_{1}$, the point of the output is $z_{2}$, the point of the perturbation maximum is $z_{m}\left(z_{1}<z_{m}<z_{2}\right)$ :

$$
z_{1}=\frac{z_{0}}{1+\cos \theta}, \quad z_{2}=\frac{\pi+z_{0}}{1+\cos \theta}, \quad z_{m}=\frac{\pi / 2+z_{0}}{1+\cos \theta} .
$$

Because of the symmetry, the deflection may happen only in the plane $(z y): \alpha_{y}$. Let us define $F_{y}(z)$ as

$$
F_{y}(z)=\left.\frac{\partial \phi_{y}}{\partial y}\right|_{y=0}, \quad \phi_{y}=\frac{1}{2} h \sin ^{2} \theta \sin \left(z-z_{0}+z \cos \theta-y \sin \theta\right),
$$

$\phi_{y}=0$ outside the $\mathrm{GW}$ pulse.

Then the deflection angle in the first part of the way within the wave is:

$$
\alpha_{1}=\int_{z_{1}}^{z_{m}} F_{y}(z) d z=-\frac{1}{2} h \frac{\sin ^{3} \theta}{1+\cos \theta}=-\frac{1}{2} h(1-\cos \theta) \sin \theta .
$$

The deflection angle in the second part of the way within the wave is:

$$
\alpha_{2}=\int_{z_{m}}^{z_{2}} F_{y}(z) d z=\frac{1}{2} h \frac{\sin ^{3} \theta}{1+\cos \theta}=\frac{1}{2} h(1-\cos \theta) \sin \theta .
$$



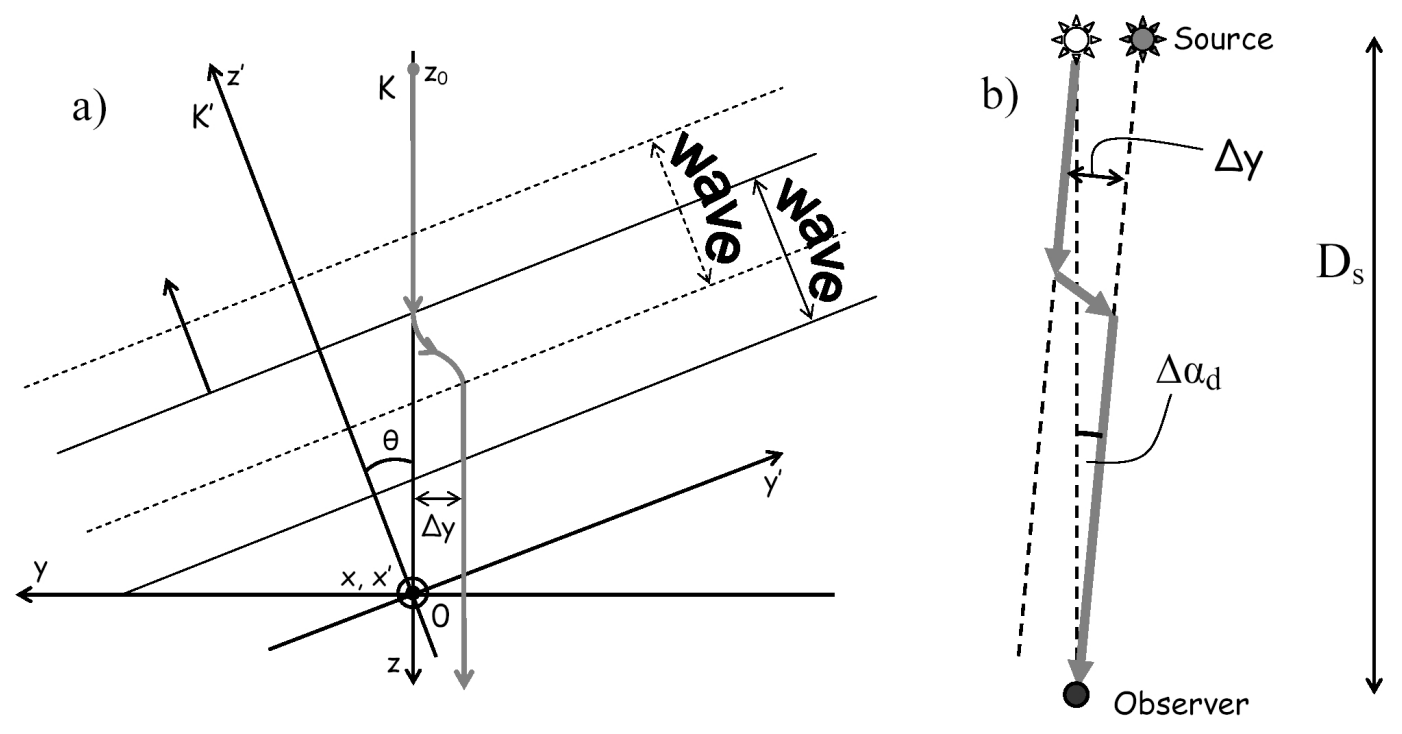

Figure 1: (a) Passing of the photon through the GW. The positions of the wave packet at the time of the photon input and the photon output are shown by full line and dashed line correspondingly. (b) The observational effect of the displacement in trajectory of the photon.

And the total deflection angle is:

$$
\hat{\alpha}=\int_{z_{1}}^{z_{2}} F_{y}(z) d z=0 .
$$

The top part of the sinusoid is symmetrical relative to the vertical axis. We also have considered a non-symmetrical plane waveform and have obtained numerically that vanishing of the deflection angle occurs in this case too.

\section{The displacement and its observational effects}

To find the displacement we need to integrate farther the equation (2.3), what gives the trajectory $y(z)$ of the photon within the wave $\left(z_{1}<z<z_{2}\right)$ :

$$
y(z)=\frac{1}{2} h \frac{\left(-1+\cos \left(z-z_{0}+z \cos \theta\right)\right) \sin ^{3} \theta}{(1+\cos \theta)^{2}}, \quad y\left(z_{1}\right)=0 .
$$

We see that the photon trajectory has a sinusoid form within wave. Using (3.1), we see that the total displacement along the axis $y$ does not vanish, and it is equal to:

$$
\Delta y=y\left(z_{2}\right)-y\left(z_{1}\right)=-h \frac{\sin ^{3} \theta}{(1+\cos \theta)^{2}}=-h \frac{1-\cos \theta}{1+\cos \theta} \sin \theta .
$$

In dimensional variables we have:

$$
\Delta y=-h \frac{\delta}{\pi} \frac{\sin ^{3} \theta}{(1+\cos \theta)^{2}} .
$$


Thus although initial and final directions of photon coincide, and the deflection angle vanishes, the displacement in the trajectory occurs (see Fig.1a). This displacement is absent in case of $\theta=0$ (the photon and the GW directions are parallel) and reaches its maximum in the case at $\theta=\pi / 2$ (the photon and the GW directions are orthogonal). It is clear that this displacement will be equal to zero if we consider the whole sinusoid with the top and the bottom parts, because the displacement due to the top part of the sinusoid will be cancelled by the displacement due to the bottom part. Therefore this displacement takes place mainly in the case of isolated wave pulses, which have a form similar to the top part of the sinusoid or when it has the non-symmetrical top and bottom parts of wave profile. The wave pulses may be produced, for example, during stellar collapse (see [6], paper contains many figures with waveforms) or during formation of large scale structure of the Universe (see [7]).

Directions of photons passing through the GW packet does not change, therefore any focusing of rays does not occur in this case. Thus the displacement in trajectories does not lead to any magnification effect. But the displacement leads to change of the angular position of object $\Delta \alpha_{d}$ for distant observer (see Fig.1b):

$$
\Delta \alpha_{d}=\frac{\Delta y}{D_{s}} \simeq \frac{h \delta}{D_{s}},
$$

where $h$ is the amplitude of the GW pulse, $\delta$ is its thickness and $D_{s}$ is a distance between the source and the observer.

Let us estimate the change of the angular position for the GW pulses produces during formation of large scale structure of the Universe in dark matter (see [7]). For estimates we put $h=10^{-11}$, $\delta=M p c, D_{s}=100 M p c$, then we obtain

$$
\Delta \alpha_{d} \simeq 2 \cdot 10^{-8} \text { arcsec }
$$

\section{Acknowledgments}

Authors are thankful to M. Barkov for useful discussions. This work was partially supported by RFBR grants 05-02-17697, 06-02-90864 and 06-02-91157, RAN Program "Formation and evolution of stars and galaxies" and Grant for Leading Scientific Schools NSh-10181.2006.2. Work of O.Yu. Tsupko was also partially supported by the Dynasty Foundation.

\section{References}

[1] V.B. Braginsky, N.S. Kardashev, A.G. Polnarev, and I.D. Novikov, Nuovo Cimento B 105 (1990) 1141.

[2] V. Faraoni, Astrophys. J. 398 (1992) 425.

[3] T. Damour, G. Esposito-Farèse, Phys. Rev. D 58 (1998) 042001.

[4] G.S. Bisnovatyi-Kogan, O.Yu. Tsupko, Gravitation and Cosmology (2008), accepted.

[5] L.D. Landau, E.M. Lifshitz, The Classical Theory of Fields, Pergamon, Oxford 1993.

[6] R.A. Saenz, S.L. Shapiro, Astrophys. J. 221 (1978) 286.

[7] G.S. Bisnovatyi-Kogan, MNRAS 347 (2004) 163. 